\title{
Finitely Generated Ideals in Rings of Analytic Functions
}

\author{
JAMES J. KeLleHER and B. A. TAYLOR
}

\section{Introduction}

Given a domain $\Omega$ of $\mathbb{C}^{n}$ and a function $p \geqq 0$ on $\Omega, A_{p}=A_{p}(\Omega)$ denotes the ring of all functions $f: \Omega \rightarrow \mathbb{C}$ analytic on $\Omega$ for which there exist constants $c_{1}=c_{1}(f) \geqq 0$ and $c_{2}=c_{2}(f) \geqq 0$ such that $|f(z)| \leqq c_{1} \exp \left(c_{2} p(z)\right)$, $z \in \Omega$. If $F_{1}, \ldots, F_{N} \in A_{p}$, and if $G \in A_{p}$ belongs to the ideal $\mathscr{F}\left(F_{1}, \ldots, F_{N}\right)$ of $A_{p}$ generated by $F_{1}, \ldots, F_{N}$, then it is easily seen that there must exist constants $c_{1}, c_{2} \geqq 0$ such that

$$
|G(z)| \leqq c_{1}\|F(z)\| \exp \left(c_{2} p(z)\right), \quad z \in \Omega,
$$

where $\|F(z)\|^{2}=\sum_{k=1}^{N}\left|F_{k}(z)\right|^{2}, z \in \Omega$. Here we investigate the extent to which the converse statement holds: that is, if Eq. (1.1) holds for some $c_{1}, c_{2} \geqq 0$, is it true that $G \in \mathscr{F}\left(F_{1}, \ldots, F_{N}\right)$ ?

For example, in [3] Hörmander showed (for suitably restricted functions $p$ ) that his $L^{2}$-estimates for solutions to the non-homogeneous Cauchy-Riemann equations could be combined with a homological argument to prove that this is the case for the function $G \equiv 1$, thereby generalizing some results of the present authors [6] for the ring $E_{0}$ of all entire functions of exponential type in one complex variable (i.e., $\Omega=\mathbb{C}^{1}$ and $p(z)=|z|$ ). He also showed there how the deep corona theorem of $\mathrm{L}$. Carleson [2], which is the corresponding statement for the ring $A_{p}=H_{\infty}$ (where we take $\Omega=\left\{z \in \mathbb{C}^{1}:|z|<1\right\}$ and $p \equiv 0$ ) could be handled in this same framework, although the principal difficulties remain the same. Further, as we show below, the converse statement is true in the event that $\mathscr{I}\left(F_{1}, \ldots, F_{N}\right)$ is a principal ideal of $A_{p}$.

In general, however, it is known that (1.1) is not a sufficient condition for $G \in \mathscr{I}\left(F_{1}, \ldots, F_{N}\right)$, as shown by Rao [8] for the case $A_{p}=H_{\infty}$. (Also, see Theorem 3.6 below.) Indeed, when $n>1$ condition (1.1) does not even imply that $G$ belongs locally to $\mathscr{I}\left(F_{1}, \ldots, F_{N}\right)$. However, using Hörmander's techniques, we show here that (again with suitable restrictions on $p$ ) the following result holds:

Theorem 1.1. There exists an integer $k \geqq 1$ such that $G^{k} \in \mathscr{I}\left(F_{1}, \ldots, F_{N}\right)$ for all $G \in A_{p}$ satisfying (1.1); in fact, we may take $k=\min \{2 n+1,2 N-1\}$.

We indicate briefly below the modifications required in the arguments of [3] to prove this theorem, and we subsequently give numerous applications to the ideal theory of the rings $A_{p}$. We also look into the "best possible" value 
of $k$ in Theorem 1.1 - in particular, we show that for the ring $E_{0}$ the "best possible" value is $k=2$. It is also shown that similar results hold in rings of analytic functions which are not necessarily of the form $A_{p}$, and in the last section we briefly describe a generalization of these methods to the case of finitely generated $A_{p}$-modules. We note, however, that the restrictions on $p$ exlude the ring $H_{\infty}$, and we do not know if analogous results hold for this ring. I. Cnop [15] has also proved Theorem 1.1 by applying the holomorphic functional calculus of $\mathrm{L}$. Waelbrock.

\section{Finitely Generated Ideals of $A_{p}$}

First we fix our notation and state the main hypotheses on the rings $A_{p}$. These will be maintained throughout this paper.

Definition 2.1. Let $\mathscr{L}$ denote the set of all complex-valued Lebesgue measurable functions on $\Omega$, identifying functions which differ only on a set of measure zero.

(1) $W_{p}=W_{p}(\Omega)$ denotes the set of all $f \in \mathscr{L}$ for which there exists a constant $C=C(f) \geqq 0$ such that

$$
\int_{\Omega}|f(z)|^{2} \exp (-C p(z)) d \mu(z)<+\infty .
$$

(Here, and for the rest of this paper, $\mu$ denotes Lebesgue measure on $\mathbb{C}^{n}$.)

(2) $B_{p}=B_{p}(\Omega)$ denotes the set of all $f \in \mathscr{L}$ for which there exists a constant $C=C(f) \geqq 0$ such that

$$
\text { ess.sup. }|f(z)| \exp (-C p(z))<+\infty .
$$

Evidently $A_{p}=B_{p} \cap A(\Omega)$, where $A(\Omega)$ denotes the set of all $f \in \mathscr{L}$ which are analytic on $\Omega$. Also, $A_{p}$ and $B_{p}$ are commutative rings (under the usual pointwise operations) which contain the constants, while $W_{p}$ is a module over the ring $B_{p}$.

In what follows we shall always suppose that $p$ is plurisubharmonic on $\Omega$ and that (i) and (ii) of [3] are satisfied. Since $p$ is then bounded above on compact subsets of $\Omega$, each $f \in W_{p}$ is locally integrable on $\Omega$ and the $\bar{\partial}$-operator acts on $W_{p}$ in the distributional sense. Now fix $F_{1}, \ldots, F_{N} \in A_{p}$, not all identically zero, and define $\|F\| \in B_{p}$ as in the previous section. Theorem (1.1) gives conditions on an element $G \in A_{p}$ which ensure that $G$ belongs to the ideal $\mathscr{I}=\mathscr{I}\left(F_{1}, \ldots, F_{N}\right)$ of $A_{p}$ generated by $F_{1}, \ldots, F_{N}$, these conditions requiring that $G$ not grow too rapidly when compared with $\|F\|$. We shall investigate such growth restrictions in more detail below, but here we simply indicate how the result follows from the methods of [3]. Our proof makes use of the Koszul complex induced by $F_{1}, \ldots, F_{N}$ (cf. [9], pp. 358-368) as previously employed by Hörmander.

Following the notation of [3], for $r \neq 0$ we let $L_{r}=L_{r}^{0}$ denote the space of all differential forms of type $(0, r)$ on $\Omega$ with coefficients in $W_{p}$. Moreover, for 
$s>0$ we let $\Gamma_{s}$ denote the set of all $s$-tuples $I=\left(i_{1}, \ldots, i_{s}\right)$ with $1 \leqq i_{1}, i_{2}, \ldots, i_{s} \leqq N$, and $L_{r}^{s}$ denotes the set of all skew-symmetric mappings from $\Gamma_{s}$ into $L_{r}$. (Equivalently, $L_{r}^{s}=\Lambda^{s}\left(\mathbb{C}^{N}\right) \otimes L_{r}$ for all $r, s \geqq 0$.) Note that $L_{r}^{s}=0$ if either $r>n$ or $s>N$. Now the $\bar{\partial}$-operator acts componentwise on the elements of $L_{r}^{s}$ and yields a linear mapping $\bar{\partial}: L_{r}^{s} \rightarrow L_{r+1}^{s}$ such that $\bar{\partial}^{2}=0$, and we obtain a double complex from

Definition 2.2. For $r, s \geqq 0$ we define $P_{F}: L_{r}^{s+1} \rightarrow L_{r}^{s}$ as follows: for $\alpha \in L_{r}^{s+1}$, $P_{F} \alpha$ is the interior product of $\alpha$ with $F=\left(F_{1}, \ldots, F_{N}\right) \in L_{0}^{1}$. That is,

$$
\left(P_{F} \alpha\right)\left(i_{1}, \ldots, i_{s}\right)=\sum_{j=1}^{N} F_{j} \alpha\left(i_{1}, \ldots, i_{s}, j\right)
$$

for all $\left(i_{1}, \ldots, i_{s}\right) \in \Gamma_{s}$. We also define $P_{F} \alpha=0$ for all $\alpha \in L_{r}^{0}$.

It is easily verified that $P_{F}^{2}=0$, whereas $\bar{\partial} P_{F}=P_{F} \bar{\partial}$ since $F_{1}, \ldots, F_{N}$ are analytic. Here we are concerned with the homological properties of this complex, and we observe that $G \in A_{p}$ belongs to $\mathscr{I}$ if and only if there exists $h \in L_{0}^{1}$ with $\bar{\partial} h=O$ and $P_{F} h=G$.

We first note that the conditions on $p$ as given in [3] imply the following results, which we state without proof. The reader is referred to [3] and [4] for further details.

Theorem 2.3. We have:

(1) $\Omega$ is a domain of holomorphy.

(2) $B_{p} \subset W_{p}$

(3) $A_{p}=\left\{f \in W_{p}: \bar{\partial} f=0\right\}=W_{p} \cap A(\Omega)$.

(4) If $f \in A_{p}$, then $\frac{\partial f}{\partial z_{k}} \in W_{p}, 1 \leqq k \leqq n$.

(5) If $r, s \geqq 0$ and $g \in L_{r+1}^{s}$ is such that $\bar{\partial} g=0$, then $g=\bar{\partial} h$ for some $h \in L_{r}^{s}$.

It is actually these facts which are of interest to us, and not the actual conditions on $p$. Our methods will have obvious application to situations in which an appropriate version of the above result holds, enabling us to obtain results for rings of analytic functions not of the form originally considered by Hörmander. Note, however, that the above conditions exclude the ring $H_{\infty}$.

Theorem 1.1 will be realized from the same proof as that of Theorem 7 of [3], except that we need the following lemma about distributional derivatives.

Lemma 2.4. Let $h_{1}, \ldots, h_{l} \in A_{p}$ and define $H(z)=\left[\left.\left|h_{1}(z)^{2}+\cdots+\right| h_{l}(z)\right|^{2}\right]^{1 / 2}$, $z \in \Omega$. Suppose that $H \neq 0$ and let $g \in L_{r}, 0 \leqq r<n$, be such that $\bar{\partial} g=0$ and $H^{-2} g \in L_{r}$. Then $\bar{\partial}\left(H^{-2} \bar{h}_{i} g\right) \in L_{r+1}$ for $1 \leqq i \leqq l$. In fact,

$$
\bar{\partial}\left(H^{-2} \bar{h}_{i} g\right)=H^{-4} \sum_{j=1}^{l} h_{j} \overline{\left(h_{j} \partial h_{i}-h_{i} \partial h_{j}\right)} \wedge g .
$$

The proof of this lemma is routine, so we omit it. Using it, however, the arguments used in obtaining Lemma 6 and Theorem 7 of [3] then yield the following propositions. 
Proposition 2.5. Let $r, s \geqq 0$ and $k \geqq 1$ be integers and let $\alpha \in L_{r}^{s}$ be such that $P_{F} \alpha=0$ and $\alpha\|F\|^{-k} \in L_{r}^{s}$. Then there exists $\beta \in L_{r}^{s+1}$ such that $P_{F} \beta=\alpha$ and $\beta\|F\|^{1-k} \in L_{r}^{s+1}$. Moreover, if $k>1$ and $\bar{\partial} \alpha=0$, then $\beta$ may be chosen so that $\delta \beta\|F\|^{2-k} \in L_{r+1}^{s+1}$.

In fact, following Hörmander, we may take $\beta=\|F\|^{-2} \alpha \wedge \bar{F}$, where $\alpha \wedge \bar{F}$ denotes the exterior product of $\alpha$ with $\bar{F}=\left(\bar{F}_{1}, \ldots, \bar{F}_{N}\right) \in L_{0}^{1}$. Specifically,

$$
\beta(I)=\|F\|^{-2} \sum_{k=1}^{s+1}(-1)^{s+1-k} \bar{F}_{i_{k}} \alpha\left(I_{k}\right),
$$

where $I=\left(i_{1}, \ldots, i_{s+1}\right) \in \Gamma_{s+1}$ and $I_{k} \in \Gamma_{s}$ is obtained from $I$ by deleting $i_{k}$, $1 \leqq k \leqq s+1$. The first part of the proposition follows as in [3], while the concluding statement is an immediate consequence of Eq. (2.1). The following result is then obtained from Proposition 2.5 by a standard diagram chase through the double complex $\left\{L_{r}^{s}\right\}$, which we omit.

Theorem 2.6. Let $\alpha \in L_{r}^{s}$ be such that $\bar{\partial} \alpha=P_{F} \alpha=0$ and suppose that $\alpha\|F\|^{-k} \in L_{r}^{s}$ for some $k \geqq \operatorname{Min}\{2(n-r)+1,2(N-s)-1\}$. Then there exists $\beta \in L_{r}^{s+1}$ with $\bar{\partial} \beta=0$ and $P_{F} \beta=\alpha$.

The special case $r=s=0$ of this result contains Theorem 1.1, for it clearly requires that $\mathscr{I}$ contain all those $G \in A_{p}$ for which $G\|F\|^{-k} \in W_{p}$ for some $k \geqq \operatorname{Min}\{2 n+1,2 N-1\}$. Also, the case $r=s=1$ yields another criterion for $G \in \mathscr{I}$ which is useful in the classification of the closed ideals of $A_{p}$ (in some natural topology - see e.g. [7], [13] or [14]).

Theorem 2.7. Let $G \in A_{p}$ and $D_{1}$ and $D_{2}$ be compact subsetts of $\Omega$ with $D_{1} \operatorname{Cint}\left(D_{2}\right)$ such that

(1) $\int_{\Omega-D_{1}}|G|^{2}\|F\|^{-2(2 n+1)} e^{-C p} d \mu<+\infty$ for some $C>0$.

(2) There exist $h_{1}, \ldots, h_{N}$ analytic on some neighborhood of $D_{2}$ with $G=\sum_{i=1}^{N} h_{i} F_{i}$ inside $D_{2}$ and

$$
\int_{D_{2}-D_{1}}\left|h_{i}\right|^{2}\|F\|^{-2(2 n+1)} d \mu<+\infty, \quad 1 \leqq i \leqq N .
$$

Then $G \in \mathscr{I}$.

Proof. Fix a $C^{\infty}$-function $\varphi$ supported on $D_{2}$ such that $\varphi(z)=1$ for all $z \in D_{1}$. We set $\gamma=\|F\|^{-2} G \wedge \bar{F}$ and define $\gamma^{\prime}$ as $\gamma_{i}^{\prime}=\varphi h_{i}+(1-\varphi) \gamma_{i}, 1 \leqq i \leqq N$, so it is clear from our hypotheses that $\gamma^{\prime} \in L_{0}^{1}$ and $P_{F} \gamma^{\prime}=G$. Moreover, with $\alpha=\bar{\partial} \gamma^{\prime}$ we have $\alpha_{i}=\left(h_{i}-\gamma_{i}\right) \bar{\partial} \varphi+(1-\varphi) \bar{\partial} \gamma_{i}, 1 \leqq i \leqq N$, and $\alpha \in L_{1}^{1}$. Indeed, we have $\alpha\|F\|^{1-2 n} \in L_{1}^{1}$ and $P_{F} \alpha=\bar{\partial} \alpha=0$, so it follows from Theorem 2.6 that $\alpha=P_{F} \beta$ for some $\beta \in L_{1}^{2}$ with $\bar{\partial} \beta=0$. If we now choose $\gamma^{\prime \prime} \in L_{0}^{2}$ such that $\bar{\partial} \gamma^{\prime \prime}=\beta$, then for $h=\gamma^{\prime}-P_{F} \gamma^{\prime \prime} \in L_{0}^{1}$ we obtain $\bar{\partial} h=0$ and $P_{F} h=G$, as desired.

In this setting we also consider the set $\mathscr{J}=\mathscr{J}\left(F_{1}, \ldots, F_{N}\right)$ of all functions $G \in A_{p}$ for which there exist constants $C_{1}=C_{1}(G) \geqq 0$ and $C_{2}=C_{2}(G) \geqq 0$ 
such that $|G(z)| \leqq C_{1}\|F(z)\| \exp \left(C_{2} p(z)\right)$ for all $z \in \Omega$. Evidently $\mathscr{I}$ is an ideal of $A_{p}$ which contains $\mathscr{I}$, and from Theorem 2.6 , we immediately obtain

Theorem 2.8. We have $\mathscr{J}^{k} C \mathscr{I}$ for all $k \geqq \operatorname{Min}\{2 n+1,2 N-1\}$. In particular, $\mathscr{J}$ and $\mathscr{I}$ have the same radical in $A_{p}$.

We remark that in the case considered by Hörmander $\|F\|$ is a unit of the ring $B_{p}$, so that division by $\|F\|$ is always possible and Lemma 2.4 is not needed. Indeed, as mentioned in the introduction, Hörmander had shown that $\mathscr{I}\left(F_{1}, \ldots\right.$, $\left.F_{N}\right)=A_{p}$ if and only if $\|F\|$ is a unit of $B_{p}$, and as a simple generalization of this we prove

Corollary 2.9. If either $\mathscr{I}$ or $\mathscr{J}$ is principal in $A_{p}$, then $\mathscr{I}=\mathscr{J}$.

Proof. First suppose that $h \in A_{p}$ generates $\mathscr{I}$ and define $f_{i}=F_{i} / h, 1 \leqq i \leqq N$, so $f_{i} \in A_{p}$ and $\|F(z)\|=|h(z)| \cdot\|f(z)\|$, where $\|f\| \in B_{p}$ is define in the obvious way. Then for any $g \in \mathscr{J}$ we have $C_{1}, C_{2} \geqq 0$ such that

$$
\left|\frac{g(z)}{h(z)}\right| \leqq C_{1}\|f(z)\| \exp \left(C_{2} p(z)\right) \leqq C_{1}^{\prime} \exp \left(C_{2}^{\prime} p(z)\right),
$$

so $h$ divides $g$ in $A_{p}$, or $g \in \mathscr{I}$, as desired. On the other hand, if $h$ generates $\mathscr{J}$ and we choose $C_{1}, C_{2} \geqq 0$ such that $|h(z)| \leqq C_{1}\|F(z)\| \exp \left(C_{2} p(z)\right)$, then we clearly have $\|f(z)\| \geqq C_{1}^{-1} \exp \left(-C_{2} p(z)\right), z \in \Omega$, so $\|f\|$ is a unit of $B_{p}$. Thus $1 \in \mathscr{I}\left(f_{1}\right.$, $\left.\ldots, f_{N}\right)$, whence $h \in \mathscr{I}$.

Finally, we note that the methods above apply to rings other than those of the form $A_{p}$. It suffices merely that the results of Theorem 2.3 remain valid for appropriately defined spaces $W$ and $B$. To be specific, consider the case $\Omega=\mathbb{C}^{n}$ and $p(z)=\lambda(\|z\|)$, where $\lambda \geqq 0$ is non-decreasing continuous function defined on $\{x \geqq 0\}$ for which the function $x \rightarrow \lambda\left(e^{x}\right)$ is convex on $-\infty \leqq x<+\infty$ (so $p$ is plurisubharmonic on $\mathbb{C}^{n}$ ). The results of 2.3 need not apply to the ring $A_{p}$ in this case, but we may successfully consider the following larger ring:

Definition 2.10. Denote by $E(\lambda)$ the ring of all entire functions $f: \mathbb{C}^{n} \rightarrow \mathbb{C}$ for which there exist constants $C_{1}, C_{2}, C_{3} \geqq 0$ such that

$$
|f(z)| \leqq C_{1} \exp \left(C_{2} \lambda\left(C_{3}\|z\|\right)\right), \quad z \in \mathbb{C}^{n} .
$$

(Rings of this type have previously been considered by Rubel and Taylor [11].) If we now define the spaces $W(\lambda)$ and $B(\lambda)$ in the obvious way, it is not difficult to verify that the corresponding statements of 2.3 are valid. In general, however, we have $A_{p} \neq E(\lambda)$, and in fact these rings coincide if and only if $\lambda$ is slowly increasing in the sense of [11]. We shall not give further details here except to state the following result.

Theorem 2.11. Given $F_{1}, \ldots, F_{N} \in E(\lambda)$, let $\mathscr{I}$ be the ideal of $E(\lambda)$ generated by $F_{1}, \ldots, F_{N}$ and let $\mathscr{J}$ be the ideal of all $G \in E(\lambda)$ for which there exist constants $C_{1}, C_{2}, C_{3} \geqq 0$ such that for all $z \in \mathbb{C}^{n}$

$$
|G(z)| \leqq C_{1}\|F(z)\| \exp \left(C_{2} \lambda\left(C_{3}\|z\|\right)\right) .
$$

Then $\mathscr{J}^{k} C \mathscr{I}$ for $k \geqq \operatorname{Min}\{2 n+1,2 N-1\}$. 
Also, instead of the ring $E(\lambda)$ one could also consider the ring of all entire functions $f: \mathbb{C}^{n} \rightarrow \mathbb{C}$ for which there exists constants $C_{1}, C_{2}, C_{3} \geqq 0$ for which

$$
|f(z)| \leqq C_{1} \exp \left(C_{2} \lambda\left(\|z\|+C_{3}\right)\right), \quad z \in \mathbb{C}^{n},
$$

and the analogue of Theorem 2.8 is valid in this ring as well.

\section{The Case $n=1$}

In this section we discuss the above results when the functions involved depend on only one complex variable. Of course even in this case one expects the algebraic structure of the rings $A_{p}$ to be more complicated than that for the full ring $A(\Omega)$, this being a rather reasonable object about which a great deal is known. (For example, see [5].) Here we give some examples of this as regards the ideals $\mathscr{I}$ and $\mathscr{J}$, and we shall obtain some improvements in the above results. In some cases the restriction to $n=1$ is essential, while elsewhere it is not, but for simplicity we do not attempt complete generality. In fact, we shall ultimately consider only the ring of entire functions of exponential type, which we denote here by $E_{0}$ (i.e., $\left.E_{0}=A_{p}(\mathbb{C}), p(z)=|z|\right)$. Here we have available the extensive classical theory of entire functions. For the remainder of this section we have $n=1$ unless specifically stated otherwise.

We first consider the problem of improving the integer $k$ of the statement $\mathscr{J}^{k} \mathrm{C} \mathscr{I}$. There is some indication that for the rings $A_{p}$ considered here the integer $k=\operatorname{Min}\{2 n+1,2 N-1\}$ of Theorem 2.8 is not the best possible, but that the result is true for $k=\operatorname{Min}\{n+1, N\}$. We are able to exhibit examples for which this is the case, but only when $n=1$ (that is, $\mathscr{J}^{2} \subset \mathscr{I}$ ). Before restricting our attention to entire functions we consider the general situation and introduce the following notation: given $F_{1}, \ldots, F_{N} \in A_{p}$, denote by $v=v\left(F_{1}, \ldots, F_{N}\right)$ the Laplacian of $\log \|F(z)\|$. Since $\log \|F(z)\|$ is subharmonic on $\Omega$, the distribution $v$ is a positive measure (c.f. Hörmander [4], page 19, and Schwartz [12], page 29). In our case we write $v=w+v$, where $w \geqq 0$ is a function defined and infinitely differentiable on the complement of the discrete set $\{z \in \Omega:\|F(z)\|=0\}$, while $v$ is a positive measure supported on this set.

Definition 3.1. We say that a finite set $\left\{F_{1}, \ldots, F_{N}\right\} \subset A_{p}$ is congenial, or simply that $F_{1}, \ldots, F_{N} \in A_{p}$ are congenial, if there exists a constant $C>0$ such that

$$
\int_{\Omega} w(z) \exp (-C p(z)) d \mu(z)<+\infty .
$$

We say that $A_{p}$ is a congenial ring if every finite subset of $A_{p}$ is congenial.

Now a short computation yields

$$
w=\|F\|^{-4} \sum_{i, j=1}^{N}\left|F_{i} F_{j}^{\prime}-F_{i}^{\prime} F_{j}\right|^{2},
$$


so evidently $\left\{F_{1}, \ldots, F_{N}\right\}$ is congenial if and only if $\|F\|^{-2}\left(F_{i} F_{j}^{\prime}-F_{i}^{\prime} F_{j}\right) \in W_{p}$, $1 \leqq i, j \leqq N$.

Theorem 3.2. If $F_{1}, \ldots, F_{N} \in A_{p}$ are congenial, then Theorem 2.8 is valid with $k=2$; that is, $\mathscr{J}^{2} \subset \mathscr{I}$.

Proof. It is evidently sufficient to show that $g \in \mathscr{I}$ for all $g \in A_{p}$ for which $g\|F\|^{-2} \in B_{p}$. Now for such a $g$ we have $g=P_{F} \alpha$, where $\alpha=\left(\alpha_{1}, \ldots, \alpha_{N}\right) \in L_{0}^{1}$ is given as $\alpha_{i}=\|F\|^{-2} g \bar{F}_{i}, 1 \leqq i \leqq N$ (c.f. Eq. (2.2)), and thus

$$
\bar{\partial} \alpha_{i}=\|F\|^{-4} g \sum_{j=1}^{N} F_{j} \overline{\left(F_{j} \partial F_{i}-F_{i} \partial F_{j}\right)},
$$

valid in view of Lemma 2.4. We (formally) write (3.3) as $\bar{\partial} \alpha=P_{F} \beta$, where

$$
\beta_{i j}=\|F\|^{-4} g \overline{\left(F_{j} F_{i}^{\prime}-F_{i} F_{j}^{\prime}\right)}, \quad 1 \leqq i, j \leqq N .
$$

Evidently $\|F\| \beta \in L_{1}^{2}$, but we claim that actually $\beta \in L_{1}^{2}$, or equivalently, $\beta_{i j} \in W_{p}$ for $1 \leqq i, j \leqq N$. This is obvious from the condition on $g$ and the hypothesis of congeniality, and since $\bar{\partial} \beta=0$, there exists $\gamma \in L_{0}^{2}$ with $\bar{\partial} \gamma=\beta$. Then $h=\alpha$ $-P_{F} \gamma \in L_{0}^{1}$ and $\vec{\partial} h=0, P_{F} h=g$, as desired.

We now show that for certain choices of $p$ the ring $A_{p}$ is congenial. Specifically, we show that this is the case for the ring $E_{0}$ of entire functions of exponential type, though other examples are readily available. We require the following simple consequence of Green's theorem for plane regions.

Lemma 3.3. For any $u \in C^{2}(\mathbb{C})$ we have

$$
\int_{0}^{r} t^{-1} S(t) d t=\frac{1}{2 \pi} \int_{0}^{2 \pi} u\left(r e^{i \theta}\right) d \theta-u(0)
$$

where

$$
S(t)=\frac{1}{2 \pi} \int_{\{|z| \leqq t\}} \int_{\mid t^{2}}\left(\frac{\partial^{2} u}{\partial x^{2}}+\frac{\partial^{2} u}{\partial y^{2}}\right) d x d y .
$$

Theorem 3.4. $E_{0}$ is a congenial ring.

Proof. We require the existence of a constant $C>0$ such that (3.1) is valid. To this end first suppose that $F_{1}, \ldots, F_{N}$ have no common zeros, so $w \in C^{\infty}(\mathbb{C})$. Choose $C_{1}, C_{2} \geqq 0$ such that $\log \|F(z)\| \leqq C_{1}|z|+C_{2}$. An application of the above lemma to $u=\log \|F\|$ gives

$$
\int_{0}^{r} t^{-1} S(t) d t \leqq 2\left(C_{1} r+C_{2}\right), \quad r \geqq 0 .
$$

Now $S$ is non-negative and increasing, since $u$ is subharmonic, so the standard argument $\int_{0}^{r} t^{-1} S(t) d t \geqq \int_{r / e}^{r} t^{-1} S(t) d t \geqq S(r / e)$ implies the existence of constants 
$C_{1}, C_{2} \geqq 0$ such that $S(r) \leqq C_{1} r+C_{2}, r \geqq 0$. Then for $C \geqq 0$,

$$
\begin{aligned}
\iint_{\mathbb{C}} w(z) e^{-C|z|} d x d y \leqq & \iint_{\{|z| \leqq 1\}} w(z) d x d y \\
& +\sum_{n \geqq 0} \iiint_{\left\{2^{n}<|z| \leqq 2^{n+1}\right\}} e^{-C|z|} d x d y \\
& \leqq\left(C_{1}+C_{2}\right)+\sum_{n \geqq 0} e^{-2^{n} C} \iint_{\left\{|z| \leqq 2^{n+1}\right\}} w(z) d x d y \\
& \leqq\left(C_{1}+C_{2}\right)+\sum_{n \geqq 0}\left(2^{n+1} C_{1}+C_{2}\right) e^{-2^{n} C} .
\end{aligned}
$$

Evidently this series converges for $C>0$ sufficiently large, as desired. For the case where $F_{1}, \ldots, F_{N}$ have common zeros, the desired conclusion may be realized via a standard limiting argument, which we omit.

As other examples we remark that the rings $E(\lambda)$ defined in the previous section are all congenial (with the appropriate modification made in the definition of congeniality). In particular, the ring of all entire functions of order $\varrho$, finite type, is congenial (i.e., the ring $A_{p}(\mathbb{C}), p(z)=|z|^{\varrho}, \varrho>0$ ). For congeniality with non-radial functions $p$ we cite the rings $A_{p}(\mathbb{C})$ with

and

$$
p(z)=|x|^{\varrho}+\log (1+|z|), \quad \varrho \geqq 1
$$

$$
p(z)=|x|^{e}+|y|^{\sigma}, \quad \varrho, \sigma \geqq 1,
$$

where $z=x+i y$. The proofs for all of these rings are essentially the same, and analogous statements hold for unions of such rings, such as the ring of all entire functions of finite order.

To obtain a substantial improvement in the value of $k$ for $n>1$ is apparently more difficult. Condition (3.1) may be replaced by an analogous condition restricting the integrability of the form $\bar{\partial} \partial \log \|F\|=\omega$, and reasoning similar to the above shows that for the ring $A_{p}\left(\mathbb{C}^{n}\right)$ with $p(z)=\|z\|$, Theorem 1.1 is valid for $k=2 n$. However, these arguments involve only the first pullback from $L_{1}^{1}$ to $L_{1}^{2}$, whereas an estimate of the pullback from $L_{2}^{2}$ to $L_{2}^{3}$ depends on the behaviour of the form $\omega \wedge \omega$. Similarly, higher powers of $\omega$ occur at later stages of the complex, and we know of no suitable estimates for these forms. We emphasize that $k$ must increase linearly with $n$, for this is the case for the ring of polynomials in $n$ complex variables (i.e., the ring $A_{p}\left(\mathbb{C}^{n}\right)$ with $p(z)$ $=\log (1+\|z\|))$.

In what follows we give several examples of the possible relationship between the ideals $\mathscr{I}$ and $\mathscr{I}$. As above, we shall consider only the case $A_{p}=E_{0}$, and we require some auxiliary results concerning this ring. These are consequences of the classical theory of entire functions, and will not be detailed here. Given $f \in E_{0}$, we define $Z(f)=\{z \in \mathbb{C}: f(z)=0\}$.

Lemma 3.5. Let $f, g \in E_{0}, g \neq 0$. Then

(1) If there exists an entire function $h$ for which $f=g h$, then $h \in E_{0}$. In particular, if $Z(g)=\emptyset$, then $1 / g \in E_{0}$. 
(2) If $Z(g)$ is infinite, then there exists $h \in E_{0}$ sich that $Z(g) \cap Z(h)=\emptyset$ and $\mathscr{I}(g, h) \neq E_{0}$.

We first show that for the ring $E_{0}$ the "best possible" value of $b>0$ in the statement "if $g \in \mathscr{J}$ then $g^{b} \in \mathscr{I}$ " is $b=2$. Here "best possible" is to be interpreted in light of Theorem 3.4 and

Theorem 3.6. For any integer $q \geqq 1$ there exist functions $F_{1}, F_{2}, g \in E_{0}$ such that

(1) g has a q-th root in $E_{0}$.

(2) $g \in \mathscr{J}\left(F_{1}, F_{2}\right)$.

(3) $g^{2-1 / q} \notin \mathscr{I}\left(F_{1}, F_{2}\right)$.

Proof. Our construction is modeled after an example of K. V. Rao [10] for the ring $H_{\infty}$. Choose $f_{1}, f_{2} \in E_{0}$ such that $Z\left(f_{1}\right) \cap Z\left(f_{2}\right)=\emptyset$ and $\mathscr{I}\left(f_{1}, f_{2}\right) \neq E_{0}$. We claim that the theorem is true with $F_{1}=f_{1}^{2 q}, F_{2}=f_{2}^{2 q}$, and $g=f_{1}^{q} f_{2}^{q}$. For (1) is obvious, while (2) follows from the inequality $2\left|a_{1} a_{2}\right| \leqq\left|a_{1}\right|^{2}+\left|a_{2}\right|^{2}$, so suppose that (3) fails. Then there exist functions $h_{1}, h_{2} \in E_{0}$ such that $g^{2-1 / q}$ $=h_{1} F_{1}+h_{2} F_{2}$, or equivalently, $f_{2}^{2 q-1}=h_{1} f_{1}+h_{2} f_{2}^{2 q} / f_{1}^{2 q-1}$. From the fact that $f_{1}$ and $f_{2}$ have no common zeros we see that $f_{1}^{2 q-1}$ divides $h_{2}$ in the ring of all entire functions, hence in the ring $E_{0}$. That is, $H_{2}=h_{2} / f_{1}^{2 q-1} \in E_{0}$, and similarly we have $H_{1}=h_{1} / f_{2}^{2 q-1} \in E_{0}$. However, obviously $H_{1} f_{1}+H_{2} f_{2}=1$, in contradiction to our original choice of $f_{1}$ and $f_{2}$, whence $g^{2-1 / q} \notin \mathscr{I}\left(F_{1}, F_{2}\right)$.

In particular, taking $q=1$ yields an example for which $\mathscr{I} \varsubsetneqq \mathscr{J}$. Also, it is not difficult to modify this example so as to obtain $\mathscr{J}^{2} \subseteq \mathscr{I} \varsubsetneqq \mathscr{J}$. Basically, one need merely observe that if $F_{1}, F_{2} \in E_{0}$ have a common zero in $\mathbb{C}$, then we always have $\mathscr{J}^{2} \subseteq \mathscr{I}$, for at any such common zero all of the elements of $\mathscr{J}^{2}$ must have a zero of appreciably higher order. We have no specific example to show that the equality $\mathscr{J}^{2}=\mathscr{I}$ can occur, and it is doubtful that this is ever the case, except when $\mathscr{J}=\mathscr{I}=E_{0}$.

Secondly, we show that there exist $F_{1}, F_{2} \in E_{0}$ for which $\mathscr{I}\left(F_{1}, F_{2}\right)=\mathscr{J}\left(F_{1}\right.$, $F_{2}$ ) but with $\mathscr{I}\left(F_{1}, F_{2}\right)$ not principal in $E_{0}$. (Compare with Corollary 2.9.)

Definition 3.7. A discrete subset $\left\{z_{k}\right\}_{k \geqq 1}$ of $\mathbb{C}$ is said to be interpolating for $E_{0}$ if the following condition is satisfied: for any sequence $\left\{b_{k}\right\}_{k \geq 1}$ of complex numbers for which there exist constants $C_{1}, C_{2} \geqq 0$ such that $\left|b_{k}\right| \leqq C_{1} e^{C_{2}\left|z_{k}\right|}$, $k \geqq 1$, there exists $g \in E_{0}$ such that $g\left(z_{k}\right)=b_{k}$ for all $k \geqq 1$.

Such sequences have been previously considered by A. F. Leont'ev [8]. In particular, every infinite discrete subset of $\mathbb{C}$ contains an infinite subset which is interpolating for $E_{0}$.

Theorem 3.8. There exists $F_{1} \in E_{0}$ such that $\mathscr{I}\left(F_{1}, \ldots, F_{N}\right)=\mathscr{J}\left(F_{1}, \ldots, F_{N}\right)$ for all $F_{2}, \ldots, F_{N} \in E_{0}$. In fact, this is the case whenever $F_{1}$ has only simple zeros and $Z\left(F_{1}\right)$ is interpolating for $E_{0}$. (For example, $F_{1}(z)=\sin z$.)

Proof. Suppose $F_{1} \in E_{0}$ has only simple zeros and that $Z\left(F_{1}\right)=\left\{z_{k}\right\}_{k \geqq 1}$ is interpolating for $E_{0}$. Let $g \in \mathscr{J}\left(F_{1}, \ldots, F_{N}\right)$, so we have $C_{1}, C_{2} \geqq 0$ such that

$$
|g(z)| \leqq C_{1}\left[\left|F_{1}(z)\right|+\cdots+\left|F_{N}(z)\right|\right] \exp \left(C_{2}|z|\right), \quad z \in \mathbb{C},
$$


and choose $a_{i j} \in \mathbb{C}, 1<i \leqq N, 1 \leqq j$, so that

$$
\left|a_{i j}\right|=1, \quad a_{i j} F_{i}\left(z_{j}\right) \geqq 0 .
$$

Define $b_{i j} \in \mathbb{C}, 1<i \leqq N, 1 \leqq j$, by

$$
b_{i j}= \begin{cases}0 & \text { if } F_{2}\left(z_{j}\right)=\cdots=F_{N}\left(z_{j}\right)=0, \\ \frac{a_{i j} g\left(z_{j}\right)}{\mid F_{2}\left(z_{j}|+\cdots+| F_{N}\left(z_{j}\right) \mid\right.} & \text { otherwise, }\end{cases}
$$

and consider the sequences $\left\{b_{i j}\right\}_{j \geqq 1}, 1<i \leqq N$. Evidently $\left|b_{i j}\right| \leqq C_{1} e^{c_{2}\left|z_{j}\right|}, j \geqq 1$, so there exist functions $h_{2}, \ldots, h_{N} \in E_{0}$ for which $h_{i}\left(z_{j}\right)=b_{i j}, 1<i \leqq N, 1 \leqq j$. Then the function $h=g-\left(h_{2} F_{2}+\cdots+h_{N} F_{N}\right)$ belongs to $E_{0}$, and $h\left(z_{j}\right)=0$ for all $j \geqq 1$, and thus $F_{1}$ divides $h$ in $E_{0}$, since $F_{1}$ has only simple zeros. We then have $h_{1}=h / F_{1} \in E_{0}$ and $g=h_{1} F_{1}+\cdots+h_{N} F_{N}$.

Corollary 3.9. There exist $F_{1}, F_{2} \in E_{0}$ such that $\mathscr{I}\left(F_{1}, F_{2}\right)=\mathscr{J}\left(F_{1}, F_{2}\right)$, but such that $\mathscr{I}\left(F_{1}, F_{2}\right)$ is not principal in $E_{0}$.

Proof. Let $F_{1} \in E_{0}$ be any function having only simple zeros such that $Z\left(F_{1}\right)$ is infinite and interpolating for $E_{0}$ and choose $F_{2} \in E_{0}$ such that $Z\left(F_{1}\right) \cap Z\left(F_{2}\right)=\emptyset$ and $\mathscr{I}=\mathscr{I}\left(F_{1}, F_{2}\right) \neq E_{0}$. Now $\mathscr{I}=\mathscr{J}\left(F_{1}, F_{2}\right)$, but $\mathscr{I}$ is not principal in $E_{0}$. For if $h \in E_{0}$ generates $\mathscr{I}$, then $h$ must be a non-unit of $E_{0}$, whence there exists $a \in \mathbb{C}$ such that $h(a)=0$, and therefore $f(a)=0$ for all $f \in \mathscr{I}$. However, by our choice of $F_{1}$ and $F_{2}$ we have either $F_{1}(a) \neq 0$ or $F_{2}(a) \neq 0$, a contradiction.

Remark. The condition that $Z(F)$ be interpolating is equivalent to $\left|F^{\prime}\left(z_{j}\right)\right|$ $\geqq \varepsilon e^{-A|z j|}$ for some $\varepsilon, A>0$, and all the zeros $z_{j}$ of $F$.

As a third example we consider the question of the maximality of ideals of the form $\mathscr{I}\left(F_{1}, \ldots, F_{N}\right)$ and/or $\mathscr{J}\left(F_{1}, \ldots, F_{N}\right)$.

Lemma 3.10. Suppose that $F_{1}, \ldots, F_{N} \in E_{0}$ have no common zeros and that $\mathscr{I}\left(F_{1}, \ldots, F_{N}\right) \neq E_{0}$. Then there exists $F_{N+1} \in E_{0}$ such that

(1) $F_{N+1} \notin \mathscr{J}\left(F_{1}, \ldots, F_{N}\right)$,

(2) $\mathscr{I}\left(F_{1}, \ldots, F_{N+1}\right) \neq E_{0}$.

Proof. It follows from our hypotheses that there exists a discrete subset $\left\{z_{k}\right\}_{k \geq 1}$ of $\mathbb{C}$ such that

$$
\left|F_{1}\left(z_{k}\right)\right|+\cdots+\left|F_{N}\left(z_{k}\right)\right| \leqq \frac{1}{k} \exp \left(-k\left|z_{k}\right|\right), \quad k \geqq 1 .
$$

If we suppose, as we may, that $\left|z_{k}\right| \leqq\left|z_{k+1}\right|$ for all $k \geqq 1$, then for any subsequence $\left\{w_{j}=z_{k j}\right\}_{j \geqq 1}$ for which $j \leqq k_{j}$ for all $j \geqq 1$ we also have

$$
\left|F_{1}\left(w_{k}\right)\right|+\cdots+\left|F_{N}\left(w_{k}\right)\right| \leqq \frac{1}{k} \exp \left(-k\left|w_{k}\right|\right), \quad k \geqq 1 .
$$

Now there exist two such disjoint subsequences $\left\{a_{k}\right\}_{k \geqq 1}$ and $\left\{b_{k}\right\}_{k>1}$ and a function $g \in E_{0}$ such that $g\left(a_{k}\right)=0$ and $g\left(b_{k}\right)=1$ for all $k \geqq 1$. (These sub- 
sequences are essentially chosen so as to be interpolating for $E_{0}$ - we omit the details.) Then with $F_{N+1}=g$ we have

$$
\left|F_{1}\left(a_{k}\right)\right|+\cdots+\left|F_{N+1}\left(a_{k}\right)\right| \leqq \frac{1}{k} \exp \left(-k\left|a_{k}\right|\right), \quad k \geqq 1,
$$

which implies $\mathscr{I}\left(F_{1}, \ldots, F_{N+1}\right) \neq E_{0}$. Further, suppose that (1) fails and let $C_{1}, C_{2} \geqq 0$ such that for all $z \in \mathbb{C}$

$$
\left|F_{N+1}(z)\right| \leqq C_{1}\left[\left|F_{1}(z)\right|+\cdots+\left|F_{N}(z)\right|\right] \exp \left(C_{2}|z|\right) .
$$

Then for $z=b_{k}$ we obtain

$$
k \exp \left(k\left|b_{k}\right|\right) \leqq C_{1} \exp \left(C_{2}\left|b_{k}\right|\right), \quad k \geqq 1,
$$

and this is absurd since $\left|b_{k}\right| \rightarrow \infty$ as $k \rightarrow \infty$, whence $F_{N+1} \notin \mathscr{J}\left(F_{1},, F_{N}\right)$.

By repeated application of this result we obtain a sequence $\left\{F_{k}\right\}_{k \geqq 1}$ of elements of $E_{0}$ with $Z\left(F_{1}\right) \cap Z\left(F_{2}\right)=\emptyset$ such that $F_{N+1} \notin \mathscr{J}\left(F_{1}, \ldots, F_{N}\right) \neq E_{0}$ for all $N \geqq 1$. This furnishes another proof of the well-known fact that $E_{0}$ is not Noetherian. Also, one may conclude that if $M$ is a maximal ideal of $E_{0}$, then either $M$ is principal or $M$ is not finitely generated. More precisely,

Theorem 3.11. If $F_{1}, \ldots, F_{N} \in E_{0}$ have no common zeros, then neither $\mathscr{I}$ nor $\mathscr{J}$ is maximal in $E_{0}$. Conversely, if either $\mathscr{I}$ or $\mathscr{J}$ is maximal, then there exists a unique point $a \in \mathbb{C}$ such that

$$
\mathscr{I}=\mathscr{J}=M_{a}=\left\{f \in E_{0}: f(a)=0\right\} .
$$

Proof. In view of the lemma above, only the converse statement remains to be proved. Now if $\mathscr{I}$ is maximal, then by the above lemma there exists $a \in \mathbb{C}$ such that $F_{1}(a)=\cdots=F_{N}(a)=0$, whence $\mathscr{I} C M_{a}$ and thus $\mathscr{I}=M_{a}, \mathscr{I}$ being maximal. This implies that $\mathscr{I}$ is principal, since $M_{a}$ is generated by $z-a$, whence $\mathscr{I}=\mathscr{J}$ by Corollary 2.9. Evidently this same argument applies in the event that $\mathscr{J}$ is maximal.

\section{Finitely Generated Modules}

In this section we briefly describe an extension of the previous results to the $A_{p}$-modules $\mathscr{M}=\left(A_{p}\right)^{m}, m \geqq 1$. The algebra required to deal with the case $m>1$ is considerably more complicated than that above, for the Koszul complex introduced in Definition 2.2 must be replaced by the generalized Koszul complex of D. Buchsbaum [1]. We do not give the details here but will state the results obtained.

Given $F_{1}, \ldots, F_{N} \in \mathscr{M}$, not all identically zero, let $F=\left(F_{i j}\right)$ denote the $m \times N$ matrix whose $j$-th column is $F_{j}, 1 \leqq j \leqq N$, and let $\mathcal{N}=\mathscr{N}\left(F_{1}, \ldots, F_{N}\right)$ be the submodule of $\mathscr{A}$ generated by $F_{1}, \ldots, F_{N}$. The matrix $F$ defines a homomorphism of $B_{p}$-modules $F:\left(W_{p}\right)^{N} \rightarrow\left(W_{p}\right)^{m}$, namely $(F \alpha)_{i}=\sum_{j=1}^{N} F_{i j} \alpha_{j}, 1 \leqq i \leqq m$, 
and the Koszul complex used above gives a free resoution of this map in the case $m=1$. The generalized Koszul complex gives a free resolution for $m \geqq 1$, and the results of the second section (specifically Theorems 2.6, and 2.7) extend to this complex. Here we consider Theorem 2.6, for which we require some new notations.

Definition 4.1. Given $I \in \Gamma_{m}$, we denote by $\Delta(I)$ the determinant of the $m \times m$ matrix whose $j$-th column is $F_{i j}, 1 \leqq j \leqq m$. Moreover, for $G \in \mathscr{M}$ and $I \in \Gamma_{m-1}, \Delta(G ; I)$ denotes the determinant of the $m \times m$ matrix whose first column is $G$ and whose $(j+1)$-th column is $F_{i j}, 1 \leqq j<m$. Finally, we define $\|F\| \in B_{p}$ as

$$
\|F\|=\left(\sum_{I \in \Gamma_{m}}|\Delta(I)|^{2}\right)^{1 / 2} .
$$

(This agrees with our earlier notation for the case $m=1$.)

Let us first suppose that $\|F\| \neq 0$. In particular, this implies that $N \geqq m$ and that the matrix $F(z)=\left(F_{i j}(z)\right)$ has rank $m$ for almost all $z \in \Omega$. In this case we have

Theorem 4.2. There exists an integer $k \geqq 1$ such that if $G \in \mathscr{M}$ and $\Delta(G ; I)\|F\|^{-k} \in W_{p}$ for all $I \in \Gamma_{m-1}$, then $G \in \mathscr{N}$. In fact, we may take $k=\operatorname{Min}\{2 n+1,2(N-m)+1\}$.

Of course, this is exactly Theorem 2.6 (with $r=s=0$ ) when $m=1$, and an analogous result holds for the modules $(E(\lambda))^{m}, m \geqq 1$. In addition, if $n=1$ and $A_{p}$ is a congenial ring, then the statement of this theorem is valid for $k=2$.

The degenerate case $\|F\| \equiv 0$, which does not occur when $m=1$, can be reduced to the non-degenerate case above in a straightforward way, as we now indicate. To be specific, suppose that the $m \times N$ matrix $F$ is such that $\|F\| \equiv 0$, let $\tilde{F}$ denote the $(m-1) \times N$ matrix obtained from $F$ by deleting the last row of $F$, and suppose that $\tilde{F}$ has rank $m-1$ at some point of $\Omega$. For any $G=\left(G_{1}, \ldots, G_{m}\right)$ $\in \mathscr{M}$ we let $\tilde{G}$ denote the $(m-1)$-tuple $\left(G_{1}, \ldots, G_{m-1}\right) \in \tilde{\mathscr{M}}=\left(A_{p}\right)^{m-1}$, and we let $\tilde{\mathscr{N}}$ be the submodule of $\tilde{\mathscr{M}}$ generated by $\tilde{F}_{1}, \ldots, \tilde{F}_{N}$. It is not difficult to establish the following (purely algebraic) fact:

Theorem 4.3. In order that $G \in \mathscr{M}$ belong to $\mathscr{N}$ it is necessary and sufficient that

(1) $\Delta(G ; I)=0$ for all $I \in \Gamma_{m-1}$, and

(2) $\tilde{G} \in \tilde{\mathscr{N}}$.

Thus the question of whether $G \in \mathscr{N}$ is reduced, modulo the algebraic conditions of (1), to that of whether $\tilde{G} \in \tilde{\mathscr{N}}$, and since our assumptions require that $\|\tilde{F}\| \neq 0$, Theorem 4.2 provides a criterion for this. Naturally, similar remarks apply when the rank of $F$ is less that $m-1$. A general result along these lines may be obtained, although we shall not state it formally here. It will imply that an element $G \in \mathscr{M}$ belongs to $\mathscr{N}$ if: first, certain algebraic equations corresponding to (1) of Theorem 4.3 are satisfied; and second, analytic conditions corresponding to the hypotheses of Theorem 4.2 hold, these being relative to a submatrix of $F$ of maximal rank. 
Finally, we observe that as an immediate consequence of Theorem 4.2 we have

Corollary 4.4. The following are equivalent:

(1) $\mathscr{N}=\mathscr{M}$,

(2) $\|F\|$ is a unit of $B_{p}$.

(3) The determinants $\Delta(I), I \in \Gamma_{m}$, generate $A_{p}$.

This is obviously the generalization of Hörmander's result to the case $m \geqq 1$. However, we emphasize that this can easily be obtained directly by a formal algebraic argument; that is, for the rings $A_{p}$, Corollary 4.4 holds for all $m \geqq 1$ if and only if it holds for $m=1$. Hence the last result is valid for the modules $\left(H_{\infty}\right)^{m}, m \geqq 1$, for when $m=1$ this exactly Carleson's corona theorem, but we do not know if Theorems 2.6, 2.8, or 4.2 are valid for $H_{\infty}$.

\section{Bibliography}

1. Buchsbaum, D.: A generalized Koszul complex, I. Trans. Amer. Math. Soc. 111, 183-196 (1964).

2. Carleson, L.: The Corona theorem. Proc. $15^{\text {th }}$ Scand. Congress, Lecture Notes in Mathematics, 118 , pp. $121-132$, Springer 1970.

3. Hörmander, L.: Generators for some rings of analytic functions. Bull. Amer. Math. Soc. 73, 943-949 (1967).

4. - An introduction to complex analysis in several variables. Princeton, New Jersey: D. van Nostrand 1966.

5. Kelleher, J.J.: Rings of meromorphic functions on non-compact Riemann surfaces. Canad. J. Math. 21, 284-300 (1969).

6. - Taylor, B.A.: An application of the Corona theorem to some rings of entire functions. Bull. Amer. Math. Soc. 73, 246-249 (1967).

7. - - Closed ideals in locally convex algebras of analytic functions, in preparation.

8. Leontev, A.F.: On entire functions of exponential type assuming given values at given points. Isv. Akad. Nauk. SSSR, Ser. Mat. 13, 33-34 (1949), (Russian) MR 10, 602 (1949).

9. Northcott, D.: Lessons on rings, modules, and multiplicities. London: Cambridge University Press 1968.

10. Rao, K.V.Rajeswara: On a generalized corona problem. J. d'Analyse Math. 18, 277-278 (1967).

11. Rubel, L., Taylor, B.A.: A Fourier series method for entire and meromorphic functions. Bull. Soc. Math. France 96, 53-96 (1968).

12. Schwartz, L.: Théorie des distributions. Paris: Hermann 1966.

13. Taylor, B. A.: Some locally convex spaces of entire functions. Proc. Symp. Pure Math., vol. 11, Entire functions and related parts of analysis. Amer. Math. Soc., 1968.

14. - A seminorm topology for some (DF)-spaces of entire functions (to appear in Duke J. Math.).

15. Cnop, I.: A theorem concerning holomorphic functions with bounded growth. Thesis, Vrije Universiteit Brussel, 1970.

Dr. J. J. Kelleher

Department of Mathematics

Univ. of Illinois

at Chicago Circle, USA
Dr. B. A. Taylor

Department of Mathematics

The University of Michigan

Ann Arbor, Mich. 48104, USA 